\title{
La política exterior alemana a partir de 1949. ¿Un persistente retraimiento?
}

\author{
León E. Bieber.
}

El tema a desarrollar no se propone incursionar en los diversos aspectos vinculados a la política exterior de la República Federal de Alemania durante el pasado medio siglo. Tampoco se hará un bosquejo de las relaciones que ha tenido el país con otras naciones o su papel en el marco de pactos regionales $u$ organismos internacionales. Los planteamientos se limitan a analizar dos cuestiones. En primer lugar; si la habitual discreción con la cual el gobierno alemán manejó sus relaciones internacionales hasta 1990 se ha mantenido a partir de la reunificación y, en caso dado, si esta reservada actitud podrá sustentarse en el futuro. Dado a que estas interrogantes están intimamente vinculadas a la orientación occidentalista que ha caracterizado a la República Federal y a que esta orientación tiene su expresión más significativa en la adhesión del país a la Unión Europea y a la Alianza Atlántica a partir de la Guerra Fría, las relaciones con estos bloques así como con países del ex-bloque socialista europeo tendrán que ocupar, necesariamente, el lugar central en las reflexiones.

La caída del Muro de Berlín en noviembre de 1989 y la reunificación de Alemania escasamente once meses más tarde, la repentina desaparición. del enfrentamiento bipolar que durante cuatro décadas había dominado las relaciones internacionales, la posición geopolítica que pasó a ocupar una Alemania geográficamente ampliada y demográficamente incrementada en el centro de Europa así como los desafíos que para aquel país conllevó la unificación crearon indefectiblemente fuertes especulaciones relativas a la continuidad de su política externa. De este modo, la cuestión alemana pasó a ocupar un lugar destacado en el contexto de las preocupaciones relativas a la constelación internacional de post Guerra Fría. Comenzó a conjeturarse si en la nueva coyuntura un mayor 
énfasis en los intereses nacionales no llevaría a que ese país abandone su tradicional recatamiento en materia de política externa, paso que podría conducir a relativizar, quizás hasta a revocar, la firme adhesión a la integración europea y al mundo occidental mantenidas hasta entonces. En síntesis, se planteaba la pregunta si Alemania no podría volver a verse tentada de dar curso a una política hegemónica que desde finales del siglo XIX hasta 1945 contribuyó decisivamente a desestabilizar el orden internacional.

En la exposición que sigue, se expondrán primero las principales características de la política externa alemana, en el período 1949-1989, para centrar luego la atención en las razones que motivaron las mencionadas preocupaciones. Seguidamente, se analizará si el manejo de aquella política por el gobierno de Bonn a partir de 1990 apunta hacia un distanciamiento del recato que ha mantenido durante cuatro décadas, justificando los temores que han cundido al respecto. Finalmente se harán algunas reflexiones respecto a la disyuntiva de preservación y mudanza con la cual se ve enfrentada actualmente Alemania en el campo de sus relaciones internacionales.

Durante la Guerra Fría, originada en las discordancias entre las potencias occidentales y la Unión Soviética respecto al código de normas y valores sobre el cual deba reconstruirse el orden mundial -particularmente el europeo- una vez derrotadas la Alemania nazi y el Japón, las coordenadas del sistema internacional se destacaron por su nitidez y persistencia. Preponderancia absoluta tuvo el enfrentamiento entre dos bloques, tanto en su fase de la alta tensión, como en el posterior período de distensión. Cada uno de estos bloques contaba con una potencia hegemónica indisputada, cuya supremacía mundial no llegó a ser cuestionada; ni siquiera por las pretensiones de liderazgo de una tercera posición mostradas por Charles de Gaulle. El temor a un holocausto termonuclear, palmariamente manifiesto durante la crisis de los misiles en Cuba a inicios de la década del 60 , condujo a un elevado grado de previsibilidad en el comportamiento de las fuerzas contestatarias. Pese a los problemas y las crisis intrabloque, cada uno de ellos gozaba de notable estabilidad. Pugnas y rivalidades entre países que no formaban parte de las alianzas lideradas por los Estados Unidos o por la Unión Soviética eran evaluadas acorde a criterios geoestratégicos derivados de la bipolaridad, o sencillamente ignorados.

En este contexto, la posición internacional de las dos Alemanias y consecuentemente sus políticas externas también eran diáfanas. 
Prácticamente a partir de la división del Reich alemán en zonas de ocupación militar en mayo de 1945 y de manera definitiva a partir de 1949, su parte occidental, o sea la República Federal de Alemania, pasó a constituir el extremo oriental para la defensa de los valores propios a una economía de mercado y a un orden político liberal-democrático, mientras que la oriental, es decir la República Democrática Alemana, se convirtió en la trinchera occidental de defensa de los intereses del bloque de países comunistas europeos. Integradas plenamente en uno y otro de los bloques, subordinadas, por ende, a las potencias mundiales hegemónicas y contando en su territorio con la mayor densidad mundial de armas nucleares sobre cuyo uso no podían decidir, las dos repúblicas no eran plenamente soberanas en materia de política externa. Consecuentemente, ésta no podía tener, sino, un bajo perfil. Este tampoco llegó a alterarse cuando a partir de inicios de la década del 70 la distensión permitió una mayor movilidad en la arena internacional a los Ministerios de Relaciones Exteriores de Bonn y de Berlín Oriental. De este modo, durante las cuatro décadas la política externa alemana fue marcada y definida por el conflicto Este-Oeste.

En el caso de la República Federal de Alemania, la Guerra Fría no sólo determinó su inserción en la Alianza Atlántica y el bajo perfil de su política externa. Condicionó otros tres elementos cardinales de su orientación internacional: sus fuertes vínculos de amistad con los Estados Unidos, su estrecha cooperación con Francia y, en base a ésta, la importancia otorgada a la integración regional de Europa Occidental. Sin embargo, sería una burda simplificación sostener que aquella política fue únicamente producto de circunstancias impuestas por factores exógenos. Ella fue, a su vez, frío cálculo de intereses propios. Bajo la conducción de Konrad Adenauer, un importante sector de las élites políticas y económicas de la República Federal comprendió lúcidamente que el proyecto de una Comunidad Europea no solamente constituía la única posibilidad de volver a poner en marcha sin mayores contratiempos el funcionamiento de las fuertes energéticas de Alemania, Francia y de los países del Benelux como requisito indispensable para la reconstrucción económica de la Europa Occidental. Aquel proyecto permitía, además, reedificar una Europa basada en la colaboración, con lo cual la desconfianza, el temor y el odio que Alemania haba suscitado en el Viejo Continente podrían ser eliminados. Para transformarse de enemigo en socio, de paria en actor internacional aceptado, 
pero ciertamente también para poder acceder a mercados, el gobierno de Bonn aceptó de buen gusto delegar parte de su soberanía en aras de la integración regional, primero en el marco de la Comunidad Europea de Carbón y Acero, luego en el de la Comunidad Económica Europea. Por las mismas razones, Adenauer optó por integrar a su país en la alianza militar atlántica. Si la adhesión de la República Federal de Alemania a la OTAN fue por un lado la solución institucional encontrada por los aliados occidentales para contar con una contribución alemana a la defensa del mundo occidental, evitando temores al resurgimiento de un militarismo de corte imperial, por el otro ella permitió el rearme del país escasamente una década después del derrumbe definitivo del Tercer Reich.

La política exterior del gobierno de Adenauer no fue únicamente producto de la Guerra Fría y de un ilustrado interés nacional. El terrible legado del Tercer Reich le otorgó un incuestionable componente moral. Demostrar al mundo que de la devastación sembrada por el totalitarismo nazi en Europa surgía una Alemania nueva, auténticamente democrática, dispuesta al arrepentimiento por los crímenes cometidos en nombre de la nación y a convivir pacíficamente con las demás naciones fue claro designio del primer Canciller de la República Federal. Esta dimensión ética se mantuvo firmemente aún después de que el país logró plena aceptación internacional y, como se planteará más adelante, no ha perdido vigencia hasta el presente.

La integración en la Comunidad Europea y en la Organización del Tratado Atlántico, la falta de plena soberana y la ausencia de ambiciones hegemónicas tuvieron secuelas sumamente afortunadas. Mientras Gran Bretaña y Francia se vieron impelidas a incurrir en ingentes gastos militares debido a sus compromisos coloniales y sus designios de grandes potencias, la República Federal de Alemania, exenta de compromisos y designios semejantes, pudo consagrar sus esfuerzos a la reconstrucción. En el correr de escasamente una década, logró convertirse en la segunda nación comercial más importante del mundo y ofrecer a sus habitantes un patrón de vida no conocido hasta entonces. Su poderío económico y su vocación pacifista le otorgaron reconocimiento y admiración internacional así como una posición no intencionada de liderazgo en la Comunidad Europea. Consecuentemente, la política externa pasó a contar rápidamente con el apoyo de prácticamente todos los estratos de la población y -exceptuando 
durante la primera mitad de la década del 70 las relaciones con los países del Bloque Oriental y particularmente con la República Democrática Alemana-, dejó de ser materia de grandes disensos entre los partidos políticos más importantes del país. Complementados sus pilares básicos con una abertura al diálogo y la cooperación con países del bloque socialista a partir de inicios de aquel decenio, ella finalmente condujo a la reunificación pacífica del país en 1990.

La opción en favor del mundo occidental es incuestionablemente uno de los hechos más descollantes de la historia alemana del último medio siglo, probablemente el de mayor trascendencia. En todo caso existe un innegable vínculo entre la asociación de la República Federal a la OTAN y a la Comunidad Europea y su orden constitutivo liberal-democrático; entre su opción por las normas y valores del mundo occidental y el fin del mito de un derrotero histórico particular, conocido por el denominado "Sonderweg".

Como se ha señalado en la parte inicial de esta exposición, fue en el lustro posterior al desmoronamiento del símbolo más visible de la Guerra Fría, el Muro de Berlín, que cundieron fuertes preocupaciones respecto a la continuidad que iría a tener la política exterior mantenida hasta entonces por Bonn. Ellas provinieron particularmente de las otras dos potencias más importantes en Europa Occidental; de Francia y de Gran Bretaña. EI temor ya se manifestó poco después de la abertura irrestricta del muro con la visita del Primer Ministros francés, François Mitterand, a la capital de la República Democrática Alemana. Su intención fue manifiesta: el simbólico acto quiso entapizar lo que François Mauriac sintió por Alemania al expresar que por amarla tanto estaba feliz de tener al mismo tiempo dos de ellas. Cuando Mitterand percibió que el desarrollo de los acontecimientos señalizaban una unificación, buscó medios para frenarla. Por su parte, Margaret Thatcher nunca dejó dudas sobre su malestar con la posibilidad de resurgimiento de un cíclope teutónico a consecuencia de la reunificación.

Más allá de sus evidentes causas históricas, estas e inquietudes parecidas emanaron, por un lado de la nueva configuración europea una vez superada la división del continente en dos bloques, por el otro debido a una serie de actitudes alemanas. Consumada la reunificación, Alemania pasó a contar con una población una vez y media más grande que la de Francia y Gran Bretaña, dos veces mayor que la de Polonia y con cinco y hasta ocho veces más habitantes que Holanda y Bélgica respectivamente. A pesar de que la 
unificación terminó por debilitar económicamente al país, esta realidad demográfica despertó suspicacias sobre la reaparición de una vieja potencia en el corazón de Europa capaz de hegemonizar su parte oriental y retomar, a partir de ahí, la política oscilante entre Oriente y Occidente ejecutada a inicios del siglo XX. Además, a comienzos de la década del 90, comenzó a vislumbrarse un importante cambio en las coordenadas geopolíticas de la Comunidad Europea. Si hasta entonces Francia fue su epicentro, un exitoso resultado de las negociaciones, entonces en curso, con pases escandinavos y con Austria para integrar el bloque y una futura adhesión de países de Europa Central y Oriental a él, que ya había sido demandada por algunos de ellos, terminaría por colocar a Alemania en esta privilegiada posición. Preocupación adicional causó el desmembramiento de la Unión Soviética, el rápido debilitamiento de Rusia y un probable relajamiento del interés norteamericano por los problemas europeos; factores que otorgarían a Alemania una posición de poder en el continente desconocida desde 1945.

El temor a una posible reedición de aspiraciones hegemónicas alemanas fue reforzado por actitudes y discursos de la propia República Federal. En diciembre de 1991, el gobierno de Bonn, por primera vez en la historia de sus relaciones diplomáticas, dio un paso absolutamente inusitado. Sin consenso previo ni con los Estados Unidos como tampoco con sus socios europeos, reconoció la soberanía de Eslovenia y de Croacia, llegando incluso a presionar a los demás países miembros de la Comunidad Europea para seguir su ejemplo. La decisión no tardó en despertar desagradables recuerdos -particularmente en Francia, una tradicional aliada de Serbiasobre la colaboración germano-croata durante la Segunda Guerra Mundial. En el correr del primer lustro de la década del 90, la pesada carga financiera de la reunificación -que llevó al Banco Central de Alemania a elevar las tasas de interés sobre el capital- y las insistentes demandas de Bonn por imponer sus criterios de estabilidad monetaria en el seno de la Unión Europea como prerequisito para introducir el Euro, obstaculizaron la transición a una segunda fase de la unificación económica y monetaria del bloque regional, prevista para inicios de 1994.

Con ello se acentuó la probabilidad de que el marco terminara por consolidarse como moneda de referencia en los mercados europeos. Paralelamente, se difundió entre expertos económicos una animosidad contra los acuerdos tomados en Maastricht, políticos plantearon la necesidad 
de repensar el carácter prioritario que tendría para Alemania el proceso de integración europea y el apoyo de la población a la misma comenzó a atenuarse. Mientras a comienzos de la década del 90 aproximadamente un $80 \%$ la favorecía, a mediados de la misma ya sólo era el 70\%. Difusión también encontró el discurso neonacionalista. Sintomático al respecto es el libro de Hans-Peter Schwarz intitulado "El Poder Central de Europa", publicado en 1994. En éste su autor plantea que el "gigante traumatizado", Alemania, debía superar, no negar, su pasado histórico y asumir decididamente su rol de potencia, constituyéndose en puente entre las regiones occidental, central y oriental de Europa para garantizar la estabilidad y el desarrollo continental. Dada su ubicación geográfica en el Viejo Continente, ella estaría sometida a leyes de gravitación geopolítica semejantes a los de la época del imperio creado por Bismarck. Este reto debería ser asumido. Versiones aún más extremas, como la de Karlheinz Weissmann, incluso llegan a rechazar la orientación occidentalista del país. Este tipo de planteamientos y las actitudes resumidas llevaron a que el Embajador francés acreditado en la República Federal declarase, en marzo de 1994, que estaban reapareciendo fantasmas del pasado. A su parecer, era preciso que Bonn explicitase claramente que nada iría a cambiar respecto a la alineación alemana en el Mundo Occidental, ya sea debido al traslado de la sede de gobierno a Berlín, a las relaciones germano-rusas o a consecuencia de la ampliación de la Unión Europea hacia el Norte y el Este europeo.

Considerando las inquietudes reseñadas y los factores que han contribuido a alimentarlas, cabe preguntar primero si a partir de 1989 se han producido realmente modificaciones en la política externa de la República Federal de Alemania. Dado el caso, se plantea la interrogante si estos cambios tienen un carácter tan substancial que el camino seguido y los compromisos asumidos en las pasadas cuatro décadas en el campo de las relaciones internacionales están siendo relegados en favor de priorizar intereses nacionales. En otros términos, si existen evidencias que Alemania está tentada por incursionar nuevamente en un "Sonderweg"1

En dos casos Bonn evidentemente se ha alejado de su habitual discreción: con sus reiterados titubeos respecto al ulterior proceso de la

\footnotetext{
"Camino propio o "especial". Nota del Editor.
} 
integración europea y al reconocer la soberanía de dos repúblicas que formaron parte de Yugoslavia. Cierto es también que a partir de 1990 la Europa oriental ha pasado a ocupar un lugar descollante en las preocupaciones de la República Federal. Cuáles las razones para este proceder?

Empezando por el aspecto señalado en último término. A diferencia de otras naciones, Alemania tiene fuertes obligaciones morales frente a países como Polonia y la República Checa a consecuencia de la política de conquista y rapiña del nacionalsocialismo, pero también debido a temores en Varsovia y Praga ante demandas restitutorias todavía no completamente acalladas por parte de asociaciones que aglutinan a ex habitantes de Silesia y del Sudetenland o a sus descendientes. Otorgar particular atención a esta situación y manejarla con suma cautela es un imperativo para la política externa de la República Federal. Imperativo que a partir del restablecimiento de la democracia en ambos países pasó a tener carácter prioritario con relación a muchos otros problemas internacionales que forman parte de la agenda internacional alemana. Como puerta de entrada del Oriente al Occidente de Europa y debido a su elevado patrón de vida, Alemania desde 1990 se ve enfrentada como ningún otro país al Oeste del río Elba con consecuencias del descalabro económico, de la miseria social, de las turbulencias políticas y, como en el caso de Yugoslavia, de guerras que hostigan a vastas regiones del centro y particularmente del este y sudeste europeo. El creciente flujo migratorio de éstas hacia la República Federal, debido precisamente a los factores señalados, ha incentivado una fuerte y desleal competencia a la mano de obra alemana (aprovechada sin escrúpulos por empresas privadas y por particulares) y ha contribuido decididamente al crecimiento de la criminalidad así como a la estructuración de redes de la mafia. Si se considera que estos problemas figuran actualmente entre los cuatro más importantes que afligen a la opinión pública, no sorprende el destacado interés que muestra Bonn por apoyar medidas para estabilizar las economías y solucionar los conflictos bélicos en el Este de Europa. En qué medida la política externa alemana respecto a esta región ha quedado condicionada por aspectos de la propia seguridad externa, lo ha expresado claramente el Ministro de Relaciones Exteriores, Klaus Kinkel, a mediados de 1998 al declarar que urgía solucionar la crítica situación en el Kosovo para evitar una nueva avalancha de fugitivos, buena parte de los cuales procuraría encontrar asilo en la República Federal. 
Con relación al caso de Eslovenia y Croacia cabe señalar que las aspiraciones independentistas de estas repúblicas llegaron a contar con fuerte simpatía de los partidos cristiano-demócrata, socialista y liberal alemanes todavía antes de producirse los primeros enfrentamientos militares en Yugoslavia. Los designios de soberanía fueron vislumbrados ante todo como una justa demanda contra el centralismo burocrático de Belgrado y un afán por establecer el pluralismo democrático y Estados de Derecho. Con ello, el principio de autodeterminación pasó a convertirse en fórmula mágica de la diplomacia alemana para solucionar las tensiones en el Estado creado por el Mariscal Tito y potencialmente quizás también para otros focos de fuertes discordias en el Este europeo. Sin prestar mayor atención a las incertidumbres que cundían en otros países de la Comunidad Europea y que sustentaba más de un político alemán respecto a la voluntad que realmente tendría Croacia de respetar los derechos de la población serbia en su territorio y al peligro que implicaban secesiones para el difícil equilibrio multiétnico yugoslavo, Bonn otorgó su reconocimiento diplomático a Liubliana y Zagreb a fines de 1991.

En cuanto a la Unión Europea, Alemania no sólo ha obstaculizado la transición a una segunda fase de la unión económica y monetaria debido a las razones ya señaladas. En marcado contraste con su habitual espíritu de compromiso para llegar a acuerdos en la Comunidad, ella a partir de fines de la década del 80 mostró una desconocida obstinación por imponer sus criterios para establecer una moneda europea. Esta actitud ha llevado a diversos enfrentamientos con los demás países de las asociación y particularmente con Francia. En marzo de 1987. O sea escasamente algo más de dos años y medio antes de la caída del Muro de Berlín, el Ministro de Relaciones Exteriores alemán Hans-Dietrich Genscher, declaró que el Sistema Monetario Europeo establecido en 1979 debía conducir a una unión monetaria.

Un año más tarde, presentó un memorándum para su materialización y para la creación de un Banco Central Europeo autónomo, capaz de garantizar la estabilidad del nuevo dinero. En junio de 1988, el Canciller alemán, Helmut Kohl declaró que la meta de su país era la unión europea, parte de la cual debía ser una moneda y una Banco Central únicos. Fue en la fase entre la abertura del Muro y la reunificación alemana que su gobierno comenzó a vacilar sobre la cuestión. Por un lado, en vista de las vehementes dudas que manifestó el Banco Federal de Alemania en cuanto a la 
receptividad que tendrían en los demás países estrictos criterios para garantizar la solidez del nuevo signo monetario, por el otro, debido a las incertidumbres que se habían difundido en el seno de la población respecto al proyecto. Esta actitud del gobierno de Bonn fue una de las principales razones que determinaron el ya mencionado malestar franco-británico, pero también el de otros miembros de la Comunidad, en cuanto a la futura orientación de la República Federal en sus relaciones internacionales. En esta constelación, Mitterand decidió resignarse a aceptar la unificación de las dos Alemanias si el gobierno de Kohl asumía firmemente el compromiso de no obstaculizar el camino hacia la implementación de la moneda europea. Una vez que París aceptó a regañadientes las condiciones de Bonn respecto a la absoluta autonomía del Banco Central Europeo y se mostró dispuesta a negociar una futura integración política del bloque regional, la cumbre realizada en Maastricht a fines de 1991 decidió introducir la nueva moneda de manera automática a partir del $1^{\circ}$ de enero de 1999 . Tomada la decisión, Alemania no cejó en plantear y replantear que únicamente países que habían cumplido estrictamente con las condiciones que garantizan la estabilidad de su moneda podían formar parte de la unión prevista. Además, consiguió que el Banco Europeo sea establecido en Frankfurt sobre el Meno y que el nombre de ECU fuese reemplazado por el de Euro. Toda esta inusitada tozudez creó fuertes resquemores anti alemanes. La República Federal fue acusada de arrogante y de no soslayar más sus ansias de poder. En la cumbre de Dublín, realizada en diciembre de 1996, el pacto de estabilidad monetaria presentado meses antes por el Ministro de Finanzas alemán motivó un serio enfrentamiento entre el presidente francés Jacques Chirac y Helmut Kohl. Cuando finalmente fue aceptado, Francia comenzó a dar la batalla por ocupar la presidencia del Banco Central Europeo y su Primer Ministro, Lionel Jospin, reiteró su demanda de una política europea conjunta, pero no de estabilidad monetaria, sino para crear fuentes de trabajo.

Los casos sucintamente expuestos de las actitudes asumidas por la República Federal de Alemania respecto a la unión monetaria, a la Europa oriental, así como a Eslovenia y Croacia denotan ciertamente su creciente articulación autónoma en el contexto de las relaciones internacionales. En el primer caso nombrado, hasta un tesón inusual por imponer sus posiciones. Pero estas realidades no permiten corroborar aseveraciones o alarmas sobre un viraje en su tradicional política externa. Si bien es cierto que las incisivas 
demandas por estabilidad monetaria no carecen de soberbia, ellas no pueden interpretarse como afán de supremacía. Responden más bien al trauma alemán con las dos grandes inflaciones que el país ha conocido en el siglo $X X$, a un difundido y no plenamente injustificado temor que el Euro carezca de la estabilidad que desde hace medio siglo tiene el marco alemán y al hecho de que éste se ha convertido en un verdadero emblema de orgullo nacional. Sintomático para su importancia en la conciencia colectiva no es tanto el uso que de ella han hecho los partidos de extrema derecha y uno con el singular nombre de "Iniciativa pro marco alemán. Nuevo Partido Liberal" durante la campaña para elegir un nuevo parlamento nacional a fines de septiembre de 1998 al enfatizar su decidida oposición a la moneda europea. Lo es sobre todo el que los grandes partidos no sólo hayan evadido realizar cualquier propaganda en favor del Euro, sino que soslayaron el tema. Aparte de la decidida lucha por imponer sus criterios respecto al nuevo signo monetario, Alemania ha homologado su acostumbrada solidaridad con la integración europea. A pesar de su crítica situación financiera debido a los costos de la reunificación, no ha forzado discusiones (como otrora, por ejemplo, Gran Bretaña) sobre su contribución al presupuesto de Bruselas y se ha abstenido de demandas por mayor representación o poder de voto en las principales instituciones comunitarias, limitándose a obtener un mayor número de diputados en el Parlamento de Estrasburgo, correspondiente a su crecido peso demográfico.

A pesar de las quejas, no sólo alemanas, por la creciente burocratización de la Unión Europea, por sus engorrosos procesos decisorios y pese al cúmulo de nuevos problemas que plantea la adhesión de cada vez más miembros, la República Federal no puede tener sino un decidido interés por dar continuidad y otorgar firmeza a la integración europea; tanto por las importantes ventajas económicas, como para asegurar la estabilidad del Viejo Continente y evitar un aislamiento político de sus vecinos occidentales. Sus logros económicos y concomitantemente su bienestar social, su estabilidad política y su integración en el mundo occidental son impensables sin su participación en aquel bloque, y éste sólo puede sobrevivir si existe un entendimiento franco-germano al respecto. No por acaso, Gerhard Schröder decidió visitar París para conversaciones con Chirac y Jospin escasamente tres días después de su victoria electoral el 27 de septiembre de 1998. 
Si bien propuestas como las de Hans-Peter Schwarz o decisiones como las tomadas respecto a Eslovenia y Croacia permiten entender las inquietudes que han surgido sobre metas que Alemania podría estar persiguiendo en Europa oriental, no existe evidencia alguna que su política en el transcurso de la última década está condicionada por aspiraciones hegemónicas en esa región o que su interés por ésta tenga como designio poder asumir una función pendular en el concierto de las grandes potencias. Ella no tiene ningún interés de dar secuencia a una política externa independiente en el Este de Europa, pero sí uno indiscutible, como ya lo ha demostrado con la República Checa, Hungría y Polonia, de integrar al mayor número de países de aquella región en la Unión Europea así como en la Organización del Tratado Atlántico Norte. Tampoco puede alegarse que intereses económicos, los cuales sin duda existen constituyen el principal cimiento para la política aquí en cuestión. Como se ha puntualizado, los esfuerzos por contribuir a afirmar estabilidad y previsibilidad en el comportamiento de los vecinos orientales están íntimamente vinculados al empeño de resguardar mejor la propia seguridad interna y no es exagerado sostener que básicamente cumplen un rol funcional a este designio. A su vez, aquella política está permeada de un fuerte componente de responsabilidad moral, como en el caso de las relaciones con la República Checa y Polonia, o de principios éticos como lo demuestra la importancia otorgada al principio de autodeterminación nacional para fundamentar el reconocimiento de la independencia de Croacia y Eslovenia. Con relación al proceder señalado en último término es menester recordar que esta excursión unilateral de la diplomacia alemana en la arena internacional no fue nada afortunada. Pecó de ingenuidad al no considerar las consecuencias que en efecto tuvo la secesión croata. Cuando ella desembocó en los encarnizados enfrentamientos en BosniaHerzegovina, Alemania por primera vez desde 1945 se vio obligada a enviar soldados a regiones fuera del área de defensa de la OTAN y buscó subsanar su aventurada decisión valiéndose nuevamente de actitudes morales. El país recibió a más refugiados de Bosnia que todo el resto de Europa en su conjunto y Bonn ha condenado la violación de derechos humanos por parte de Croacia contra poblaciones serbias y musulmanas. La desavenencia que esta condena provocó en las relaciones germano-croatas, la falta de influencia alemana para contribuir a la solución de los problemas en la ex Yugoslavia y el interés 
de Zagreb por firmar contratos con Francia para la construcción de una red de autopistas y con los Estados Unidos para adquirir aviones subrayan la dificultad de discernir las metas económicas y políticas que a final de cuentas ha perseguido Alemania al reconocer la independencia de Croacia.

A partir de lo expuesto cabe constatar que en el correr de la última década la República Federal no se ha apartado substancialmente de su tradicional política externa. Conjeturarlo en la importancia otorgada a sus relaciones con el Este de Europa o en su decidida actitud con referencia a la unión monetaria distorsiona la realidad. Si se busca establecer un rasgo eminentemente característico de aquella política, éste, en cualquier caso, es otro. En la parte final de la exposición se aludirá al mismo y se esbozará la situación que actualmente enfrenta el país en sus relaciones internacionales.

Nada ha distinguido tanto a la política exterior alemana desde la reunificación y la recuperación de plena soberanía externa como su finalidad de conservar la amistad con las demás naciones, particularmente con las europeas. Manifestación palmaria al respecto es el apoyo que da por un lado al deseo de Polonia y de otros países del ex bloque socialista de formar parte de la OTAN y de conservar, por el otro, las buenas relaciones con Rusia, la cual reiteradamente ha manifestado su franca oposición a todo ensanchamiento del Tratado Atlántico Norte hacia la parte oriental del Viejo Continente. Alemania es consciente que los proyectos de donación mundial del nacionalsocialismo la convirtieron en principal víctima de la Guerra Fría y que ella fue la gran ganadora cuando ésta finalizó. De esta experiencia histórica emana su señalada actitud y su vacilación de asumir delicados compromisos internacionales de imprevisibles consecuencias. De ah también su resistencia a arrogarse responsabilidad mundial o a liderar. Ella claramente prefiere adherir a decisiones de organismos internacionales o participar de acuerdos y coaliciones multilaterales.

¿Es que conseguirá mantener este recatamiento? La Guerra Fría corresponde al pasado. Con su conclusión desaparecieron los principios axiomáticos que confinaron las relaciones internacionales a partir del segundo lustro de la década del 40 . Sus parámetros vigentes durante cuatro decenios han dado paso a confusión y nuevas incertidumbres. Regiones enteras de Europa del Este han sumergido en miseria económica y social, así como en caos político. En las más diversas latitudes arrecian guerras y guerras civiles, en las cuales se yuxtaponen factores socio-económicos, 
étnicos, culturales, religiosos e ideológicos y que en varios casos han llevado a terribles genocidios. A las tendencias de globalización se contraponen nacionalismos, más o menos aguerridos, en busca de autoidentificación. Estas realidades favorecen una descontrolada difusión de armas bacteriológicas, químicas y nucleares. A la bipolaridad ha seguido una multipolaridad en la cual, aparte de una única potencia hegemónica, existen polos de poder demográfico, económico y/o militar. Los desafíos reales y potenciales que presenta esta nueva constelación en muchos casos no consiguen ser enfrentados eficazmente ni por procederes unilaterales 0 acuerdos bilaterales, como tampoco por organismos internacionales. Los sanguinarios conflictos en Africa y, todavía mucho más cerca de la frontera alemana, en la región del Cáucaso y sobre todo en el ex y en la actual Yugoslavia, la recurrente inestabilidad financiera internacional, problemas ecológicos, las crecientes amenazas de accidentes nucleares, la proliferación de mafias que trafican con drogas o con material radioactivo, tardan demasiado en o no encuentran solución por la falta de concepciones y acuerdos para combatirlos. Al igual que después de finalizada la Segunda Guerra Mundial, la esperanza que una vez concluida la Guerra Fría el mundo sería más pacífico y el futuro más promisor se ha revelado como gran ilusión.

Alemania ciertamente no es el, ni siquiera uno de los muchos acuciantes problemas internacionales. Pero son precisamente éstos, los que hacen indefectible que ella asuma mayor responsabilidad internacional, ya sea debido a su potencial económico o a su ubicación geopolítica. Importantes actores internacionales, curiosamente también Francia y Gran Bretaña, ya demandaron reiteradas veces su mayor presencia y compromiso para solucionar conflictos regionales, imputándole que no puede continuar limitándose a una diplomacia del cheque, como aconteció durante la guerra del Golfo en la fase inmediatamente posterior a la reunificación. Washington insiste que la República de Berlín debe dejar de esconderse detrás de la historia alemana como pretexto para limitar su apoyo a contribuciones económicas en caso de conflictos. Bill Clinton incluso ha sugerido un liderazgo germano-norteamericano cooperativo (una "cooperation in leadership"); propuesta que ciertamente creó resquemor en Europa. Tanto aquellos que temen por un nuevo "camino propio", como los que aseguran que éste no se reproducirá y hasta preferirían que la República Federal continuase destacándose únicamente como nación comercial y potencia 
civil, convergen en un punto. En la coyuntura de post Guerra Fría Alemania dudosamente conseguirá mantener el bajo perfil tradicional de su política externa. Ella necesariamente tendrá que transitar de su cómoda y afortunada pasividad a un rol activo de coorganizadora a nivel internacional, hecho que indefectiblemente incluye una función de bisagra en el esbozo de una nueva arquitectura europea. Para el coloso en el corazón de Europa parece haber llegado indefectiblemente aquella hora prevista hace muchos años por Golo Mann; aquella en la cual su peso político pasa a equipararse con el económico. Con sus 80 millones de habitantes la integración de Alemania en el mundo occidental facilita y fortalece su capacidad de influencia en otras regiones, especialmente al Este de su frontera, en tanto que su peso en esta región acrecienta sus posibilidades para formular e implementar futuras políticas tanto en la Unión Europea, como en la OTAN y en la Unión Europea Occidental. El arte de la diplomacia alemana en los próximos años seguramente consistirá en mantener su habitual discreción y moderación sin caer en abstinencia, en actuar con tacto sin claudicar ante responsabilidades y riesgos cuando intereses nacionales y/o los de sus tradicionales aliados están en juego. En enero de 1999 entrará en vigencia la unión económica y monetaria europea. Al mismo tiempo, Alemania asumirá por seis meses la presidencia de la Unión Europea, así como por un año la del Grupo de los Siete y de la Unión Occidental Europea. Durante su mandato en la Unión Europea ésta discutirá la "Agenda 2000" que incluye álgidos temas sobre importantes reformas estructurales. A fines de abril del año mencionado, se celebrará el cincuentenario de la OTAN, momento para el cual está prevista la adhesión de la República Checa, Hungría y Polonia a este bloque militar. Al cerrarse el siglo, la política externa alemana tendrá más de una oportunidad para responder a las expectativas cada vez mayores que en ella se centran para participar activamente, pero sin prepotencia, en la solución tanto de problemas regionales europeos como de desafíos globales. 